New Book Received *

\title{
Electronic Warfare Target Location Methods, Second Edition. Edited by Richard A. Poisel, Artech House, 2012; 422 pages. Price: £99.00, ISBN 978-1-60807-523-2
}

\author{
Shu-Kun Lin \\ MDPI AG, Kandererstrasse 25, CH-4057 Basel, Switzerland; E-Mail: lin@mdpi.com \\ Received: 10 January 2013 / Accepted: 14 January 2013 / Published: 17 January 2013
}

The following paragraphs are reproduced from the website of the publisher [1].

Describing the mathematical development underlying current and classical methods of geolocating electronic systems that are emitting, this newly revised and greatly expanded edition of a classic Artech House book offers practical guidance in electronic warfare target location. The Second Edition features a wealth of additional material including new chapters on time delay estimation, direction finding techniques, and the MUSIC algorithm. This practical resource provides you with critical design information on geolocation algorithms, and establishes the fundamentals of existing algorithms as a launch point for further algorithm development. You gain an in-depth understanding of key target location methods that you can effectively apply to your work in the field. You discover triangulation algorithms that offer a highly efficient way to geolocate targets when the real estate on the sensor systems is adequate to support an antenna array. The book also presents quadratic geolocation techniques that can be implemented with extremely modest antennas - frequently a single dipole or monopole. Moreover, this authoritative volume details methods for geolocating the source of high frequency signals with a single sensor site.

\section{Table of Contents}

\section{Preface xv}

\section{Chapter 1 Introduction to Emitter Geolocation}
1.1 Introduction
1.2 Gradient Descent Algorithm
1.3 Concluding Remarks

References 


\section{Chapter 2 Triangulation}

2.1 Introduction

2.2 Basic Concepts

2.3 Least-Squares Error Estimation

2.4 Total Least-Squares Estimation

2.5 Least-Squares Distance Error PF Algorithm

2.5.1 Brown's Least-Squares Triangulation Algorithm

2.5.2 Hemispheric Least-Squares Error Estimation Algorithm

2.5.3 Pages-Zamora Least-Squares

2.5.4 Total Least-Squares Error

2.6 Minimum Mean-Squares Error Estimation

2.6.1 Dynamical Systems

2.6.2 Linear Minimum Mean-Squares Estimation

2.6.3 Target Location Estimation with the Linear Model

2.6.4 Kalman Filter Methods

2.7 The Discrete Probability Density Method

2.8 Generalized Bearings

2.9 Maximum Likelihood PF Algorithm

2.9.1 Maximum Likelihood Estimation Triangulation Algorithm

2.9.2 Maximum Likelihood Estimation Algorithm Comparison

2.10 Multiple Sample Correlation

2.11 Bearing-Only Target Motion Analysis

2.12 Sources of Error in Triangulation

2.12.1 Geometric Dilution of Precision in Triangulation

2.12.2 LOB Error

2.12.3 Effects of Bias on Bearing-Only PF

2.12.4 Combining Noisy LOB Measurements

2.12.5 Effects of Navigation Error

2.13 Concluding Remarks

References

Appendix 2A Least-Squares Error Estimation Program Listing

Appendix 2B Generalized Bearing Program Listing

\section{Chapter 3 DF Techniques}

\subsection{Introduction}

3.2 Array Processing Direction of Arrival Measurement Methods

3.2.1 Introduction

3.2.2 The Model

3.2.3 Array Covariance Modeling

3.2.4 Direction of Arrival

3.2.5 Subspace-Based Methods

3.2.6 Beamforming AOA Estimation 
3.2.7 Maximum Likelihood AOA Estimation

3.2.8 Least-Square Error AOA Estimation

3.2.9 Decoupling Sample Source Signals from AOA Parameters

3.2.10 Gram-Schmidt Orthogonalization

3.2.11 Nonlinear Programming

3.3 Other Methods of Estimating the AOA

3.3.1 Phase Interferometry

3.3.2 Amplitude Systems

3.3.3 Doppler Direction Finder

3.4 MSE Phase Interferometer

3.4.1 Introduction

3.4.2 The Algorithm

3.4.3 Simulation

3.5 DF with a Butler Matrix

3.5.1 Introduction

3.5.2 Beamforming Network

3.5.3 Summary

3.6 Phase Difference Estimation Using SAW Devices

3.6.1 Introduction

3.6.2 SAW Characteristics

3.7 Concluding Remarks

References

Chapter 4 MUSIC

4.1 Introduction

4.2 MUSIC Overview

4.3 MUSIC

4.3.1 The MUSIC Algorithm

4.4 Performance of MUSIC in the Presence of Modeling Errors

4.4.1 Model Errors

4.4.2 Error Expressions

4.4.3 Results

4.5 Determining the Number of Wavefields

4.6 Effect of Phase Errors on the Accuracy of MUSIC

4.6.1 Introduction

4.6.2 Accuracy

4.6.3 Solutions for Errors

4.6.4 Statistics

4.6.5 Horizontal Planar Arrays

4.6.6 Simulations

4.6.7 Summary

4.7 Other Superresolution Algorithms 
4.7.1 Maximum Likelihood Method

4.7.2 Adaptive Angular Response

4.7.3 Thermal Noise Algorithm

4.7.4 Maximum Entropy Method

4.7.5 Comparisons

4.8 Concluding Remarks

References

\section{Chapter 5 Quadratic Position-Fixing Methods}

5.1 Introduction

5.2 TDOA Position-Fixing Techniques

5.2.1 Introduction

5.2.2 TDOA

5.2.3 Calculating the PF with TDOAs

5.2.4 Nonlinear Least-Squares

5.2.5 TDOA Measurement Accuracy

5.2.6 TDOA PFs with Noisy Measurements

5.2.7 TDOA Dilution of Precision

5.2.8 Bias Effects of TDOA PF Estimation

5.2.9 Effects of Movement on TDOA PF Estimation

5.3 Differential Doppler

5.3.1 Introduction

5.3.2 DD

5.3.3 DD Measurement Accuracy

5.3.4 Maximum Likelihood DD Algorithms

5.3.5 Cross-Ambiguity Function

5.3.6 Estimating the DD of a Sinusoid in Noise Using Phase Data

5.3.7 Effects of Motion on DD PF Estimating

5.4 Range Difference Methods

5.4.1 Introduction

5.4.2 Least-Squared Range Difference Methods

5.4.3 Range Difference Using Feasible Bivectors

5.5 Concluding Remarks

References

\section{Chapter 6 Time Delay Estimation}

6.1 Introduction

6.2 System Overview

6.3 Cross Correlation

6.3.1 Error Analysis of the Cross-Correlation Method

6.3.2 Flat Noise Spectra: Arbitrary Signal Spectrum

6.3.3 Flat Noise Spectra: Flat Signal Spectrum 
6.4 Generalized Cross-Correlation

6.5 Estimating the Time Delay with the Generalized Correlation Method

6.5.1 Roth Process

6.5.2 Smoothed Coherence Transform (SCOT)

6.5.3 Phase Transform (PHAT)

6.5.4 Eckart Filter

6.5.5 Maximum Likelihood

6.5.6 Variance of the Delay Estimators

6.6 Time Delay Estimation Using the Phase of the Cross-Spectral Density

6.6.1 Introduction

6.6.2 Data Model

6.6.3 Properties of the Sample CSD

6.6.4 TDOA Estimation

6.6.5 Cramer-Rao Bound

6.6.6 Other Considerations

6.6.7 Summary

6.7 Effects of Frequency and Phase Errors in EW TDOA Direction-Finding Systems

6.7.1 Introduction

6.7.2 Perfect Synchronization

6.7.3 Errors in Synchronization

6.7.4 Effects of Finite Sample Time

6.7.5 White Noise Signal

6.7.6 Simulation Results

6.7.7 Estimator Performance

6.7.8 Ramifications

6.7.9 Summary

6.8 Concluding Remarks

References

\section{Chapter 7 Single-Site Location Techniques}

7.1 Introduction

7.2 HF Signal Propagation

7.2.1 Ionograms

7.2.2 Magnetic Field Effects

7.3 Single-Site Location

7.4 Passive SSL

7.5 Determining the Reflection Delay with the Cepstrum

7.6 MUSIC Cepstrum SSL

7.7 Earth Curvature

7.8 Skywave DF Errors

7.8.1 Introduction

7.8.2 Magnetic Field Effects 


\subsubsection{Ross Curve}

7.8.4 Bailey Curve

7.9 Ray Tracing

7.9.1 Parabolic Modeling

7.10 Accuracy Comparison of SSL and Triangulation for Ionospherically Propagated Signals

7.10.1 Introduction

7.10.2 Spherical Model

7.10.3 Plane Model

7.10.4 Comparison Between SSL and Triangulation

7.10.5 Summary

7.11 Concluding Remarks

References

\section{Appendix A Grassmann Algebra}

A.1 Background

A.2 Introduction

A.3 Exterior Product

A.3.1 Properties of the Exterior Product

A.3.2 m-Vectors

A.4 Regressive Product

A.4.1 Unions and Intersections of Spaces

A.4.2 Properties of the Regressive Product

A.4.3 The Common Factor Axiom

A.4.4 The Common Factor Theorem

A.4.5 The Intersection of Two Bivectors in a 3-D Space

A.5 Geometric Interpretations

A.5.1 Points and Vectors

A.5.2 Sums and Differences of Points

A.5.3 Lines and Planes

A.5.4 Intersection of Two Lines

A.6 The Complement

A.6.1 The Complement as a Correspondence Between Spaces

A.6.2 The Euclidean Complement

A.6.3 The Complement of a Complement

A.6.4 The Complement Axiom

A.7 The Interior Product

A.7.1 Inner Products and Scalar Products

A.7.2 Calculating Interior Products

A.7.3 Expanding Interior Products

A.7.4 The Interior Product of a Bivector and a Vector

A.8 Concluding Remarks

References 


\section{Appendix B Nonlinear Programming Algorithms}
B.1 Introduction
B.2 Steepest Descent
B.2.1 Introduction
B.2.2 Method of Steepest Descent
B.2.3 Convergence
B.2.4 Scaling
B.2.5 Extensions
B.3 Gauss-Newton Method
B.4 Levenberg-Marquardt Algorithm
B.4.1 Introduction
B.4.2 Nonlinear Least-Squares Minimization
B.4.3 LM as a Blend of Gradient Descent and Gauss-Newton Iteration
B.5 Concluding Remarks

\section{References}

Acronyms

Symbols

\section{About the Author}

\section{Index}

* Editor's Note: The brief summary and the contents of the books are reported as provided by the authors or the publishers. Authors and publishers are encouraged to send review copies of their recent books of potential interest to readers of Sensors to the Publisher (Dr. Shu-Kun Lin, Multidisciplinary Digital Publishing Institute (MDPI), Kandererstrasse 25, CH-4057 Basel, Switzerland. Tel. +41-61-683-77-34; Fax: +41-61-302-89-18; E-Mail: lin@mdpi.com). Some books will be offered to the scholarly community for the purpose of preparing full-length reviews.

\section{Note}

1. The website for this book is: http://www.artechhouse.com/International/Books/Introduction-toModern-EW-Systems-1958.aspx.

(C) 2013 by the authors; licensee MDPI, Basel, Switzerland. This article is an open access article distributed under the terms and conditions of the Creative Commons Attribution license (http://creativecommons.org/licenses/by/3.0/). 